\title{
ON THE SEMIGROUP OF DIFFERENTIABLE MAPPINGS
}

\author{
SADAYUKI YAMAMURO
}

(Received 9 April 1969)

\section{To Bernhard Hermann Neumann on his 60th birthday}

Communicated by G. B. Preston

The purpose of this paper is to improve a result in [2] on the automorphisms of the semigroup $\mathscr{D}=\mathscr{D}(E)$ of all (Fréchet)-differentiable mappings of a real Banach space $E$ into itself.

We denote the derivative of $f \in \mathscr{D}$ at $a \in E$ by $f^{\prime}(a)$. This means that $f^{\prime}(a) \in \mathscr{L}=\mathscr{L}(E)$ (the Banach algebra of all continuous linear mappings of $E$ into itself with the usual upper bound norm) and

where

$$
\lim _{\|x\| \rightarrow 0}\|x\|^{-1}\|r(f ; a, x)\|=0
$$

$$
r(f ; a, x)=f(a+x)-f(a)-f^{\prime}(a)(x) \quad \text { for } x \in E .
$$

It is well-known that, for $f g$ which is defined by

$$
(f g)(x)=f(g(x)) \quad \text { for every } x \in E,
$$

we have $f g \in \mathscr{D}$ whenever $t \in \mathscr{D}$ and $g \in \mathscr{D}$, and

$$
(f g)^{\prime}(a)=f^{\prime}(g(a)) g^{\prime}(a) \text {. }
$$

This product defines a semigroup structure in $\mathscr{D}$. An automorphism $\phi$ of $\mathscr{D}$ is a bijection of $\mathscr{D}$ such that

$$
\phi(f g)=\phi(f) \phi(g) \quad \text { for every } f \in \mathscr{D} \text { and } g \in \mathscr{D} .
$$

An automorphism $\phi$ is said to be inner if there exists a bijection $h \in \mathscr{D}$ such that $h^{-1} \in \mathscr{D}$ and

$$
\phi(f)=h f h^{-1} \quad \text { for every } f \in \mathscr{D} .
$$

We denote the set of real numbers by $\mathscr{R}$. For $\alpha \in \mathscr{R}$, the mapping $x \rightarrow \alpha x$ of $E$ into itself is obviously continuous and linear. We denote this mapping by $\alpha$. Since $\alpha \in \mathscr{D}$, for an automorphism $\phi$ of $\mathscr{D}$, we can consider $\{\phi(\alpha) \mid \alpha \in \mathscr{R}\}$ which is a one-parameter group of diffeomorphisms (i.e. bijective and bi-differentiable mappings). 
For $a=\phi(0)(0)$ and the translation $t_{a}: x \rightarrow x+a$, the mapping $\phi_{0}: \mathscr{D} \rightarrow \mathscr{D}$ defined by

$$
\phi_{0}(f)=t_{a}^{-1} \phi(f) t_{a}
$$

is an automorphism which satisfies $\phi_{0}(0)=0$.

Definition. An automorphism $\phi$ of $\mathscr{D}$ is said to be uniform if, for any positive $\varepsilon \in \mathscr{R}$ and every $\left\{\alpha_{n}\right\} \subset \mathscr{R}$ such that $\alpha_{n} \rightarrow 0$, there exists a positive $\delta \in \mathscr{R}$ such that $\|x\|<\delta$ implies

$$
\sup _{u \geqq 1}\left\|\alpha_{n}^{-1} \phi_{0}\left(\alpha_{n}\right)(x)-x\right\| \leqq \varepsilon\|x\| .
$$

The main result of this paper is the following theorem.

THEOREM. An automorphism of $\mathscr{D}$ is inner if and only if it is uniform.

If $\phi(\alpha) \in \mathscr{L}$ for every $\alpha \in \mathscr{R},\{\phi(\alpha)\}$ is a one-parameter group of topological linear isomorphisms of $E$ into itself. The continuity with respect to the parameter (see (2) below) leads to the conclusion that $\phi(\alpha)=\alpha$ for every $\alpha \in \mathscr{R}$, from which the uniformity immediately follows and, therefore, $\phi$ is inner. This is the result obtained in [2].

If we take the sum $f+g$ as well as product $f g$ into consideration, the set $\mathscr{D}$ is a near-ring. If $\phi$ is a near-ring automorphism, then it is easy to see that $\phi(\alpha)=\alpha$ for every $\alpha \in \mathscr{R}$, which implies that $\phi$ is uniform. This implies that the near-rings $\mathscr{D}\left(E_{1}\right)$ and $\mathscr{D}\left(E_{2}\right)$ are isomorphic if and only if the Banach spaces $E_{1}$ and $E_{2}$ are diffeomorphic. On the other hand, from our theorem it follows that the semigroups $\mathscr{D}\left(E_{1}\right)$ and $\mathscr{D}\left(E_{2}\right)$ are isomorphic by a uniform isomorphism if and only if $E_{1}$ and $E_{2}$ are diffeomorphic.

We believe that the answer to the following problem is affirmative.

PROBLEM. Is every automorphism of $\mathscr{D}$ uniform?

Therefore, in the proof of sufficiency, we shall avoid using the uniformity wherever possible, which sometimes makes the proof unnecessarily long.

\section{Proof of the necessity}

We assume that $\phi$ is an inner automorphism of the semigroup $\mathscr{D}$. Therefore, there exists a diffeomorphism $h: E \rightarrow E$ such that $(*)$ is true. Then, since $\phi(1)=1$, we have $\left(h_{0}^{-1}\right)(0)=h_{0}^{\prime}(0)^{-1}$ and $h_{0}(0)=0$ where $h_{0}=t_{a}^{-1} h$ with $a=h(0)$.

Let $\varepsilon$ be an arbitrary positive number. There exists $\varepsilon_{1}>0$ such that

$$
\left\|h_{0}^{\prime}(0)\right\| \varepsilon_{1}+\left(\left\|h_{0}^{\prime}(0)^{-1}\right\|+\varepsilon_{1}\right) \varepsilon_{1}<\varepsilon
$$

Putting $r_{1}(x)=r\left(h_{0} ; 0, x\right)$ and $r_{2}(x)=r\left(h_{0}^{-1} ; 0, x\right)$, we can take $\delta_{1}>0$ 
such that $0<\|x\|<\delta_{1}$ implies $\left\|r_{i}(x)\right\|<\varepsilon_{1}\|x\|(i=1,2)$. Since $e_{0}^{-1} h$ is continuous, there exists $\delta>0$ such that

$$
0<\delta<\delta_{1} \text { and }\left\|h_{0}^{-1}(x)\right\|<\delta_{1} \text { if }\|x\|<\delta .
$$

Then, for $\alpha \in \mathscr{R}$ such that $0<|\alpha|<1$, if $\| x||<\delta$,

$$
\begin{aligned}
\left\|\alpha^{-1} r_{1}\left(\alpha h_{0}^{-1}(x)\right)\right\| & \leqq\left\|h_{0}^{-1}(x)\right\|\left(\left\|\alpha h_{0}^{-1}(x)\right\|\right)^{-1}\left\|r_{1}\left(\alpha h_{0}^{-1}(x)\right)\right\| \\
& <\left(\left\|h_{0}^{\prime}(0)^{-1}\right\|\|x\|+\left\|r_{2}(x)\right\|\right) \varepsilon_{1} .
\end{aligned}
$$

Therefore, since

$$
\alpha^{-1} \phi_{0}(\alpha)(x)-x=h_{0}^{\prime}(0) r_{2}(x)+\alpha^{-1} r_{1}\left(\alpha h_{0}^{-1}(x)\right),
$$

we have, if $\|x\|<\delta$,

$$
\begin{aligned}
\left\|\alpha^{-1} \phi_{0}(\alpha)(x)-x\right\| & \leqq\left\|h_{0}^{\prime}(0)\right\|\left\|r_{2}(x)\right\|+\left\|\alpha^{-1} r_{1}\left(\alpha h_{0}^{-1}(x)\right)\right\| \\
& <\left\|h_{0}^{\prime}(0)\right\| \varepsilon_{1}\|x\|+\left(\left\|h_{0}^{\prime}(0)^{-1}\right\|\|x\|+\left\|r_{2}(x)\right\|\right) \varepsilon_{1} \\
& \leqq\left\{\left\|h_{0}^{\prime}(0)\right\| \varepsilon_{1}+\left(\left\|h_{0}^{\prime}(0)^{-1}\right\|+\varepsilon_{1}\right) \varepsilon_{1}\right\}\|x\| \\
& <\varepsilon\|x\| .
\end{aligned}
$$

\section{Proof of the sufficiency}

Let $\phi$ be an automorphism of $\mathscr{D}$. The following fact has been proved by K. D. Magill, Jr. [1].

There exists a bijection $h: E \rightarrow E$ which satisfies (*).

All we know about this $h$ at this stage is that it is a bijection (i.e., one-to-one and onto). We are going to prove that $h \in \mathscr{D}$ and $h^{-1} \in \mathscr{D}$.

Since $\phi^{-1}(f)=h^{-1} f h$ and $\phi^{-1}$ is also an automorphism, any statement about $h$ can be replaced by the same statement about $h^{-1}$. We shall use this fact freely.

Moreover, we can assume that $h(0)=0$, because, if $h(0)=a \neq 0$, we have only to consider the bijection $h_{0}=t_{a}^{-1} h$, which corresponds to the automorphism $\phi_{0}$.

For the sake of convenience, we denote the set of all sequences $\left\{\varepsilon_{n}\right\} \subset \mathscr{R}$ such that $\lim _{n \rightarrow \infty} \varepsilon_{n}=0$ by $\left(c_{0}\right)$.

(1) $\inf _{n \geqq 1}\left\|\varepsilon_{n}^{-1} h\left(\varepsilon_{n} a\right)\right\|>0$ for every $a \in E$ and any $\left\{\varepsilon_{n}\right\} \in\left(c_{0}\right)$.

Assume that there exist $a \in E$ and $\left\{\varepsilon_{n}\right\} \in\left(c_{0}\right)$ such that

$$
\lim _{n \rightarrow \infty}\left\|\varepsilon_{n}^{-1} h\left(\varepsilon_{n} a\right)\right\|=0 \text {. }
$$

For any $\left\{\delta_{n}\right\} \in\left(c_{0}\right)$, taking one of its subsequences if necessary, we can assume that $\delta_{n} \varepsilon_{n}^{-1} \rightarrow 0$. Then,

$$
\delta_{n}^{-1} h\left(\delta_{n} a\right)=\delta_{n}^{-1} h\left(\delta_{n} \varepsilon_{n}^{-1} \varepsilon_{n} a\right)=\delta_{n}^{-1} \phi\left(\delta_{n} \varepsilon_{n}^{-1}\right) h\left(\varepsilon_{n} a\right) .
$$


On the other hand, the uniformity implies that there exists $\delta>0$ such that $\|x\|<\delta$ implies

$$
\sup _{n \geqq 1}\left\|\delta_{n}^{-1} \varepsilon_{n} \phi\left(\delta_{n} \varepsilon_{n}^{-1}\right)(x)\right\| \leqq\|x\| .
$$

Since $\lim _{n \rightarrow \infty} h\left(\varepsilon_{n} a\right)=0$, we get

which implies

$$
\begin{aligned}
\left\|\delta_{n}^{-1} h\left(\delta_{n} a\right)\right\| & =\mid \varepsilon_{n}^{-1}\left\|\delta_{n}^{-1} \varepsilon_{n} \phi\left(\delta_{n} \varepsilon_{n}^{-1}\right) h\left(\varepsilon_{n} a\right)\right\| \\
& \leqq\left\|\varepsilon_{n}^{-1} h\left(\varepsilon_{n} a\right)\right\|,
\end{aligned}
$$

Therefore,

$$
\lim _{n \rightarrow \infty} \delta_{n}^{-1} h\left(\delta_{n} a\right)=0 \text {. }
$$

$$
\lim _{\varepsilon \rightarrow 0} \varepsilon^{-1} h(\varepsilon a)=0,
$$

which means that $h$ is Gateaux-differentiable at 0 , because, for any $x$, if we take $\chi \in \mathscr{L}$ such that $\chi(a)=x$,

$$
\varepsilon^{-1} h(\varepsilon x)=\varepsilon^{-1} h(\varepsilon \chi(a))=\varepsilon^{-1} \phi(\chi) h(\varepsilon a),
$$

from which it follows that $\lim _{\varepsilon \rightarrow 0} \varepsilon^{-1} h(\varepsilon x)=0$. Moreover, $h$ is Gateauxdifferentiable at every point, because, for $t_{x}: z \rightarrow x+z$, we have $t_{x} \in \mathscr{D}$ and

$$
\varepsilon^{-1}[h(x+\varepsilon z)-h(x)]=\varepsilon^{-1}\left[\phi\left(t_{x}\right) h(\varepsilon z)-\phi\left(t_{x}\right) h(0)\right],
$$

from which it follows that

$$
\lim _{\varepsilon \rightarrow 0} \varepsilon^{-1}[h(x+\varepsilon z)-h(x)]=\phi\left(t_{x}\right)^{\prime}(0)\left(\lim _{\varepsilon \rightarrow 0} \varepsilon^{-1} h(\varepsilon z)\right) .
$$

If we denote the Gateaux-derivative of $h$ at $x$ by $h^{*}(x)$, then we have $h^{*}(x)=0$ for every $x \in E$. The mean value theorem then implies that $h=0$, which is a contradiction.

For the conjugate space $\bar{E}$, the value of $\bar{a} \in \bar{E}$ at $x \in E$ is denoted by $\langle x, \bar{a}\rangle$.

(2) For any $\bar{a} \in \bar{E},\langle h(x), \bar{a}\rangle$ is continuous with respect to $x$.

To prove the continuity at $a \in E$, we use the method used by K. D. Magill, Jr. [1]. We take positive $\varepsilon \in \mathscr{R}$ and non-zero $b \in E$ and consider the mapping $g \in \mathscr{D}$ such that

$$
g(x)=\beta(\langle x-h(a), \tilde{a}\rangle) b+h(a),
$$

where $\beta: \mathscr{R} \rightarrow \mathscr{R}$ is a differentiable function such that

$$
\beta(\xi)=0 \text { if }|\xi| \geqq \varepsilon ;=1 \text { if } \xi=0 .
$$

We take $f \in \mathscr{D}$ such that $\phi(f)=g$. Then, $f(a) \neq a$, because, if $f(a)=a$, we have

$$
h(a)=h f(a)=\phi(f) h(a)=g h(a)=b+h(a),
$$


which is a contradiction. Since $f$ is continuous, there exists $\delta>0$ such that $\|x-a\|<\delta$ implies $f(x) \neq a$. Therefore, if $\|x-a\|<\delta$, we have $g h(x)=$ $h f(x) \neq h(a)$, which means that $\beta(\langle h(x)-h(a), \tilde{a}\rangle) \neq 0$. By the definition of $\beta$, we have $\langle h(x)-h(a), \vec{a}\rangle<\varepsilon$.

(3) $\sup _{n \geqq 1}\left\|\varepsilon_{n}^{-1} h\left(\varepsilon_{n} a\right)\right\|<\infty$ for any $a \in E$ and any $\left\{\varepsilon_{n}\right\} \in\left(c_{0}\right)$. As a special case, $\lim _{n \rightarrow \infty} h\left(\varepsilon_{n} a\right)=0$.

Let us suppose that there exist $a \in E$ and $\left\{\varepsilon_{n}\right\} \in\left(c_{0}\right)$ such that

$$
\lim _{n \rightarrow \infty}\left\|\varepsilon_{n}^{-1} h^{-1}\left(\varepsilon_{n} a\right)\right\|=\infty \text {. }
$$

Then, for some $\bar{a} \in \bar{E}$, we have

$$
\lim _{n \rightarrow \infty}\left\langle\varepsilon_{n}^{-1} h^{-1}\left(\varepsilon_{n} a\right), \bar{a}\right\rangle=\infty .
$$

For these $a \in E$ and $a \in \vec{a}$, we consider the mapping $a \otimes \bar{a} \in \mathscr{L}$ that is defined by

Then,

$$
a \otimes \bar{a}(x)=\langle x, \bar{a}\rangle a .
$$

$$
\begin{aligned}
\phi(a \otimes \bar{a})^{\prime}(0)(a)= & \lim _{n \rightarrow \infty} \varepsilon_{n}^{-1} \phi(a \otimes \bar{a})\left(\varepsilon_{n} a\right) \\
= & \lim _{n \rightarrow \infty} \varepsilon_{n}^{-1} h\left[\left\langle h^{-1}\left(\varepsilon_{n} a\right), \bar{a}\right\rangle a\right] \\
= & \lim _{n \rightarrow \infty}\left(\varepsilon_{n}^{-1}\left\langle h^{-1}\left(\varepsilon_{n} a\right), \bar{a}\right\rangle\right)\left(\left\langle h^{-1}\left(\varepsilon_{n} a\right), \bar{a}\right\rangle\right)^{-1} \\
& \times h\left[\left\langle h^{-1}\left(\varepsilon_{n} a\right), \bar{a}\right\rangle a\right],
\end{aligned}
$$

from which it follows that

$$
\lim _{n \rightarrow \infty}\left(\left\langle h^{-1}\left(\varepsilon_{n} a\right), \bar{a}\right\rangle\right)^{-1} h\left[\left\langle h^{-1}\left(\varepsilon_{n} a\right), \bar{a}\right\rangle a\right]=0,
$$

which contradicts the facts proved in (1) and (2).

(4) For any $a \in E$ and any $\left\{\varepsilon_{n}\right\} \in\left(c_{0}\right)$, there exists a subsequence $\left\{\varepsilon_{n_{\mathbf{k}}}\right\}$ such that

is convergent.

$$
\left\{\varepsilon_{n_{k}}^{-1} h\left(\varepsilon_{n_{k}} a\right)\right\}
$$

Since $a$ can be supposed to be non-zero, we can take $\bar{a} \in \bar{E}$ such that $\langle a, \bar{a}\rangle \neq 0$ and $\phi(a \otimes \bar{a})^{\prime}(0)(a) \neq 0$. For this $a \otimes \bar{a}$, we take $\left\{\delta_{n}\right\} \in\left(c_{0}\right)$ such that

$$
\left\langle h^{-1}\left(\delta_{n} a\right), \tilde{a}\right\rangle=\varepsilon_{n},
$$

which is possible because of (2). Since the sequence of real numbers

$$
\left\{\delta_{n}^{-1}\left\langle h^{-1}\left(\delta_{n} a\right), \bar{a}\right\rangle\right\}
$$

is bounded, it contains a convergent subsequence

$$
\left\{\delta_{n_{k}}^{-1}\left\langle h^{-1}\left(\delta_{n_{k}} a\right), \bar{a}\right\rangle\right\}
$$


Then,

$$
\begin{aligned}
0 & \neq \phi(a \otimes \tilde{a})^{\prime}(0)(a)=\lim _{k \rightarrow \infty} \delta_{n_{k}}^{-1} \phi(a \otimes \tilde{a})\left(\delta_{n_{k}} a\right) \\
& =\lim _{k \rightarrow \infty} \delta_{n_{k}}^{-1}\left\langle h^{-1}\left(\delta_{n_{k}} a\right), \bar{a}\right\rangle \varepsilon_{n_{k}}^{-1} h\left(\varepsilon_{n_{k}} a\right),
\end{aligned}
$$

which implies that

$$
\lim _{k \rightarrow \infty} \delta_{n_{k}}^{-1}\left\langle h\left(\delta_{n_{k}} a\right), \bar{a}\right\rangle \neq 0 .
$$

Therefore, we have the limit

$$
\lim _{k \rightarrow \infty} \varepsilon_{n_{k}}^{-1} h\left(\varepsilon_{n_{k}} a\right)=\left(\lim _{k \rightarrow \infty} \delta_{n_{k}}^{-1}\left\langle h^{-1}\left(\delta_{n_{k}} a\right), \bar{a}\right\rangle\right)^{-1} \phi(a \otimes \bar{a})^{\prime}(0)(a) .
$$

(5) The limit $\lim _{\varepsilon \rightarrow 0} \varepsilon^{-1} h(\varepsilon a)$ exists.

We have only to show that, if the limits

$$
\lim _{n \rightarrow \infty} \varepsilon_{n}^{-1} h\left(\varepsilon_{n} a\right)=a_{1} \text { and } \lim _{n \rightarrow \infty} \delta_{n}^{-1} h\left(\delta_{n} a\right)=a_{2}
$$

exist for $\left\{\varepsilon_{n}\right\} \in\left(c_{0}\right)$ and $\left\{\delta_{n}\right\} \in\left(c_{0}\right)$, then we have $a_{1}=a_{2}$.

We can assume, taking a subsequence of $\left\{\delta_{n}\right\}$ if necessary, that

Then,

$$
\lim _{n \rightarrow \infty} \delta_{n} \varepsilon_{n}^{-1}=0 \text {. }
$$

$$
\begin{aligned}
\delta_{n}^{-1} h\left(\delta_{n} a\right) & =\delta_{n}^{-1} h\left(\delta_{n} \varepsilon_{n}^{-1} \varepsilon_{n} a\right)=\delta_{n}^{-1} \phi\left(\delta_{n} \varepsilon_{n}^{-1}\right) h\left(\varepsilon_{n} a\right) \\
& =\varepsilon_{n}^{-1}\left[\delta_{n}^{-1} \varepsilon_{n} \phi\left(\delta_{n} \varepsilon_{n}^{-1}\right) h\left(\varepsilon_{n} a\right)-h\left(\varepsilon_{n} a\right)\right]+\varepsilon_{n}^{-1} h\left(\varepsilon_{n} a\right)
\end{aligned}
$$

The uniformity then implies that

$$
\begin{aligned}
\left\|a_{2}-a_{1}\right\| & =\lim _{n \rightarrow \infty}\left\|\delta_{n}^{-1} h\left(\delta_{n} a\right)-\varepsilon_{n}^{-1} h\left(\varepsilon_{n} a\right)\right\| \\
& =\lim _{n \rightarrow \infty}\left\|\varepsilon_{n}^{-1}\left[\delta_{n}^{-1} \varepsilon_{n} \phi\left(\delta_{n} \varepsilon_{n}^{-1}\right) h\left(\varepsilon_{n} a\right)-h\left(\varepsilon_{n} a\right)\right]\right\|=0 .
\end{aligned}
$$

We denote the limit $\lim _{\varepsilon \rightarrow 0} \varepsilon^{-1} h(\varepsilon a)$ by $h^{*}(0)(a)$.

(6) $h$ is differentiable at every point in all directions.

Let $a$ be an arbitrary point and consider the mapping $t_{a}: x \rightarrow x+a$. Then, $t_{a} \in \mathscr{D}$ and

$$
\begin{aligned}
\varepsilon^{-1}[h(a+\varepsilon x)-h(a)] & =\varepsilon^{-1}\left[\phi\left(t_{a}\right) h(\varepsilon x)-\phi\left(t_{a}\right) h(0)\right] \\
& =\varepsilon^{-1}\left[\phi\left(t_{a}\right)^{\prime}(0) h(\varepsilon x)+r\left(\phi\left(t_{a}\right) ; 0, h(\varepsilon x)\right)\right] .
\end{aligned}
$$

Therefore,

$$
\lim _{\varepsilon \rightarrow 0} \varepsilon^{-1}[h(a+\varepsilon x)-h(a)]=\phi\left(t_{a}\right)^{\prime}(0) h^{*}(0)(x) .
$$

We denote this limit by $h^{*}(a)(x)$. Obviously,

$$
h^{*}(a)(\alpha x)=\alpha h^{*}(a)(x) .
$$


(7) For any $a \otimes \bar{a}, h(a \otimes \bar{a}) \in \mathscr{D}$ and

Since

$$
(h(a \otimes \bar{a}))^{\prime}(x)(y)=\langle y, \bar{a}\rangle h^{*}(\langle x, \bar{a}\rangle a)(a) .
$$

$$
\begin{aligned}
\varepsilon^{-1}[h(a \otimes \bar{a})(x+\varepsilon y)-h(a \otimes \bar{a})(x)] \\
=\varepsilon^{-1}[h(\langle x, \bar{a}\rangle a+\varepsilon\langle y, \bar{a}\rangle a)-h(\langle x, \bar{a}\rangle a)],
\end{aligned}
$$

it follows from (6) that the limit as $\varepsilon \rightarrow 0$ exists and it is

$$
\langle y, \bar{a}\rangle h^{*}(\langle x, \bar{a}\rangle a)(a),
$$

which is obviously continuous and linear with respect to $y$. Moreover,

$$
\begin{aligned}
& \varlimsup_{\|y\| \rightarrow 0}\|y\|^{-1}\|h(a \otimes \bar{a})(x+y)-h(a \otimes \bar{a})(x)-(h(a \otimes \bar{a})) *(x)(y)\| \\
& \leqq\|\bar{a}\| \varlimsup_{\|y\| \rightarrow 0} \|(\langle y, \bar{a}\rangle)^{-1}[h(\langle x, \bar{a}\rangle a+\langle y, \bar{a}\rangle a)-h(\langle x, \bar{a}\rangle a] \\
& \quad-h^{*}(\langle x, \bar{a}\rangle a)(a) \| \\
&= 0,
\end{aligned}
$$

which means that $h(a \otimes \bar{a}) \in \mathscr{D}$.

(8) For any $a \otimes \bar{a},(a \otimes \bar{a}) h \in \mathscr{D}$ and

$$
((a \otimes \bar{a}) h)^{\prime}(x)(y)=\left\langle h^{*}(x)(y), \bar{a}\right\rangle a .
$$

By (7), we have

$$
(a \otimes \bar{a}) h=\phi^{-1}(h(a \otimes \bar{a})) \in \mathscr{D} .
$$

The formula for $((a \otimes \bar{a}) h)^{\prime}(x)(y)$ is obvious.

(9) $h^{*}(a) \in \mathscr{L}$ for every $a \in E$.

The linearity follows immediately from (8). To prove the continuity, let us take an arbitrary non-zero $b \otimes \bar{b}$. Then

$$
\begin{aligned}
\left|\left\langle h^{*}(a)(x), \bar{b}\right\rangle\right| & =\|b\|^{-1}\left\|((b \otimes \bar{b}) h)^{\prime}(a)(x)\right\| \\
& \leqq\|b\|^{-1}\left\|((b \otimes \bar{b}) h)^{\prime}(a)\right\|\|x\|,
\end{aligned}
$$

which means the set

$$
\left\{h^{*}(a)(x) \mid\|x\| \leqq 1\right\}
$$

is weakly bounded. Therefore, $h^{*}(a)$ is continuous.

We define $r_{1}(x)$ and $r_{2}(x)$ by

$$
h(x)-h^{*}(0)(x)=r_{1}(x) \text { and } h^{-1}(x)-\left(h^{-1}\right) *(0)(x)=r_{2}(x) .
$$

(10) For any sequence $\left\{x_{n}\right\}$ such that $\lim _{n \rightarrow \infty} x_{n}=0$, the sequence $\left\{\left\|x_{n}\right\|^{-1} r_{i}\left(x_{n}\right)\right\}$ converges weakly to 0 for $i=1,2$. Therefore, the sequence $\left\{\left\|x_{n}\right\|^{-1} h\left(x_{n}\right)\right\}$ is bounded, which implies that $\lim _{n \rightarrow \infty} h\left(x_{n}\right)=0$. 
From (8) it follows that $(a \otimes \bar{a}) r_{1}(x)$ is the remainder of $(a \otimes \bar{a}) h$ at 0 . Therefore,

which implies that

$$
\lim _{n \rightarrow \infty}\left\|x_{n}\right\|^{-1}(a \otimes \bar{a}) r_{1}\left(x_{n}\right)=0,
$$

for every $\bar{a} \in \bar{E}$.

$$
\lim _{n \rightarrow \infty}\left\langle\left\|x_{n}\right\|^{-1} r_{1}\left(x_{n}\right), \bar{a}\right\rangle=0
$$

(11) $\lim _{\|x\| \rightarrow 0}\|x\|^{-1} r_{i}(x)=0(i=1,2)$. Therefore, $h \in \mathscr{D}$ and $h^{-1} \in \mathscr{D}$. Assume that there exists a sequence $\left\{x_{n}\right\} \subset E$ such that

$$
\lim _{n \rightarrow \infty} x_{n}=0 \text { and }\left\|x_{n}\right\|^{-1}\left\|r_{1}\left(x_{n}\right)\right\| \geqq \gamma>0 \quad(n=1,2, \cdots)
$$

for some positive $\gamma \in \mathscr{R}$. By (5), we can take $\left\{\varepsilon_{n}\right\} \in\left(c_{0}\right)$ such that

$$
\left\|\varepsilon_{n}^{-1} r_{1}\left(\varepsilon_{n} x_{n}\right)\right\| \leqq\left\|x_{n}\right\|^{2} \quad(n=1,2, \cdots) .
$$

Then, for large $n$, we have

$$
\begin{aligned}
& \left\|\varepsilon_{n}^{-1} \phi\left(\varepsilon_{n}\right) h\left(x_{n}\right)-h\left(x_{n}\right)\right\|=\left\|\varepsilon_{n}^{-1} h\left(\varepsilon_{n} x_{n}\right)-h\left(x_{n}\right)\right\| \\
& =\left\|\varepsilon_{n}^{-1} r_{1}\left(\varepsilon_{n} x_{n}\right)-r_{1}\left(x_{n}\right)\right\| \geqq\left\|r_{1}\left(x_{n}\right)\right\|-\left\|\varepsilon_{n}^{-1} r_{1}\left(\varepsilon_{n} x_{n}\right)\right\| \\
& \geqq\left(\gamma-|| x_{n} \|\right)|| x_{n}\left\|\geqq\left(\gamma-\left\|x_{n}\right\|\right)\left(\inf _{n \geqq 1}\left\|x_{n}\right\|\left\|h\left(x_{n}\right)\right\|^{-1}\right)\right\| h\left(x_{n}\right) \| .
\end{aligned}
$$

Since, by (10), inf $f_{n \geqq 1}\left\|x_{n}\right\|\left\|h\left(x_{n}\right)\right\|^{-1}>0$ which implies that $\lim _{n \rightarrow \infty} h\left(x_{n}\right)=0$, this contradicts the uniformity.

\section{References}

[1] K. D. Magill, Jr., Automorphisms of the semigroup of all differentiable functions', Glasgow Math. Journ. 8 (1967) 63-66.

[2] S. Yamamuro, 'A note on semigroups of mappings on Banach spaces', Journ. Australian Math. Soc. 9 (1969) 455-464.

Australian National University and

State University of New York at Buffalo 